\title{
Critical reevaluation of methods of recording and assessing c-VEMPS
}

\begin{abstract}
The cervical Vestibular Evoked Myogenic Potential (c-VEMP) investigates otolith and vestibular nerve function. Dysfunction is characterized by feeling similar to being on a boat or an elevator. It may be a spinning sensation when people say that they are dizzy. The c-VEMP is a short-latency electromyographic (EMG) potential and it is evoked in response to high-level acoustic stimuli. The responses are mediated by the vestibular system. Clinical applications go beyond dizziness. Defining normative data is a first and crucial step to before implementing a new technique in the clinic for applications in patients.
\end{abstract}

Keywords: vestibular, dizziness, diagnosis, potential
Volume 10 Issue 5 - 2018

\author{
Lilian Felipe \\ Assistant Professor, Lamar University, USA
}

Correspondence: Lilian Felipe, Assistant Professor, Lamar University, USA, Email Ifelipe@lamar.edu

Received: July II, 2018 | Published: September 06, 2018

\section{Introduction}

The cervical Vestibular Evoked Myogenic Potential (c-VEMP) is generally assumed to provide information about a different part of the vestibular system compared to the more routinely applied rotation, head impulse and caloric tests and can provide valuable additional diagnostic information. ${ }^{1}$ Defining normative data is a first and crucial step to before implementing a new technique in the clinic for applications in patients.

In most papers different protocols were used in those days describing various stimulation types (tone burst, clicks etc), stimulus intensities and frequencies, head and body positions, electrode positions, data-acquisition and signal processing, and output parameters like latencies, threshold and amplitude and symmetry of the responses.

In the following discussion it is presented in details the optimal protocol to apply in this test, according current literature.

\section{Stimulation type}

Various types of stimuli have been used to evoke c-VEMP responses. They include air and bone-conducted tone bursts, airconducted clicks, forehead taps, and galvanic stimulation (shortduration transmastoid direct current stimulation). ${ }^{2-5}$

A click stimulus is a block function containing a wide spectrum of frequencies. A short tone burst (STB) stimulus can be described as a short pure tone, predominantly containing one specific stimulus frequency. Clicks and STB are conducted through the middle ear and are still believed to activate - among others - saccular hair cells via movement of the endolymph near the oval window by the stapes. Bone conducted stimuli produce a wave of vibration that bypasses the middle ear and directly activate the vestibular apparatus on both sides simultaneously. Galvanic stimulation is assumed to act upon the distal part of the primary vestibular afferents. Fore head taps are shown also to be effective in eliciting c-VEMP's. However, like bone-conducted stimulation, galvanic stimulation and fore head taps, induce worse signal to noise ration as compared to air-conducted sound stimulation. Bone conduction stimulation is limited as the current available stimulators and provide still limited and often sub-threshold stimuli intensities, between 60-70 dBnHL. ${ }^{6,7}$

STB evoked C-VEMPs and click-evoked responses can be induced with good signal to noise ration, but STB require lower absolute stimulus intensities. ${ }^{8,9}$ Based on these findings we choose for the STB type as a relatively easy and effective stimulus in the routine clinical application for C-VEMP's and this is still the case at current in most clinics worldwide.

\section{Stimulus intensity and frequency}

The c-VEMP can be induced in animals by tone burst stimuli ranging from 100 to $3200 \mathrm{~Hz}$ with a greater sensitiveness towards the lower frequencies. ${ }^{10-12}$ The low frequency sensitivity in humans is even more pronounced maybe due to the larger otoconia mass in the human sacculus, compared to that in smaller animals such as a cat. ${ }^{10,13}$

Remarkably, in our hands, the responses we obtained with 1000 $\mathrm{Hz}$ toneburst were more clear than with $500 \mathrm{~Hz}$ tone burst. As it is not in line with the literature this could at least partly be due to the limitations of our equipment used those days.

At lower amplitudes and at $500 \mathrm{~Hz}$ we observed no clear and reproducible responses in quite a number of healthy subjects. At current specific equipment have been developed for c-VEMP which provide even a response with a better signal to noise ratio even when using much less intense stimulus amplitudes of $95 \mathrm{dBnHL}$ at various frequencies. The advantage of these lower amplitudes is a better comfort for patients; less fatigue while still good responses can be obtained. It is applied as an advice - in line with the current opinion and in case of using c-VEMP amplitudes as output parameters - the use of $95 \mathrm{dBnHL}$ tonebursts at $500 \mathrm{~Hz}$ for routine application.

\section{Head and body positions}

A constant muscular tension is a prerequisite to a reliable recording of c-VEMP. If the muscle is not contracted sufficiently, the c-VEMP responses may be absent. ${ }^{8}$ The response can be measured on several neck muscles, also. So both electrode position and contraction state of the muscle are crucial. Colebath et al., ${ }^{15}$ described a valid method recording response from the sternocleidomastoid (SCM) muscle and proposed this as optimal.

The many different methods of SCM activation make it difficult to compare findings across studies and thus to determine the optimal VEMP test protocol. The various methods used to obtain a tonic contraction of the SCM muscle throughout C-VEMP recording include: 1) lifting the head off the support surface to bilaterally activate the SCM muscles while in supine position (supine elevation method) ${ }^{16}$ 2) pressing the fore head against a padded bar, ${ }^{17,18}$ pushing 
with their jaw against the hand-held inflated cuff to generate specific cuff pressures, ${ }^{19}$ pressing the chin against an adjustable stand, ${ }^{20}$ or squeezing a rubber ball placed between the chin and the manubrium, ${ }^{21}$ to bilaterally activate the SCM muscles while in the sitting position (sitting forward flexion method), 3) rotating the head to the side opposite the stimulated ear to unilaterally activate the SCM on the side of the test ear while sitting, ${ }^{22-24}$ (sitting rotation method) or supine, ${ }^{25}$ (supine rotation method) positions, and 4) simultaneously lifting and rotating the head to the side opposite the stimulated ear to unilaterally activate the SCM on the side of the test ear while in the supine position, ${ }^{26}$ (supine elevation rotation method). Few studies have compared different methods of SCM activation to determine those positions that yield the most robust and reliable VEMP responses. ${ }^{25-27}$

When the c-VEMP data were collected in our studies, the patient was seated with the head turned in the opposite direction of the neck muscle contraction to be induced and the stimulated labyrinth. At that time, it was not completely clear how latencies and amplitudes were affected by different head and body positions.

Nowadays, conclude based in the current literature that head and body position significantly affected the amplitude of the VEMP, but had no significant effect on latency. Although there is still no consensus all over the world regarding the optimal head-body position, there is a tendency in favor of using the supine position with the head in flexion without rotation but with visual feedback to control muscle contraction for routine application.

\section{Electrode position}

Electrode position is another variable in recording the c-VEMP. Sheykholeslami et al., ${ }^{11}$ sought to determine the optimal electrode sites. The optimal ground electrode was considered to be at the fore head, and the reference (indifferent) electrode was positioned on the sternum. ${ }^{10}$ Like indicated in the introduction of this thesis, results from the Sheykholeslami study suggested that the active electrode placed on either the upper or middle portion of the SCM produced the clearest waveforms with largest amplitudes. This electrode configeration became more or less the standard. ${ }^{24,28,29}$

\section{Electromyography (EMG) level}

C-VEMP can be considered as a modulation of an existing tonic neck muscle contraction. The c-VEMP amplitude was shown and is known to increase with tonic muscle contraction. ${ }^{30}$ So, it is necessary to define the contraction state of the neck muscle to standardize the method and to overcome the large variation in c-VEMP amplitudes when muscle contraction is not kept constant during the measurements. To achieve a constant EMG level and thus comparable physiological conditions, a manual or automatic feedback mechanism is needed. Several devices monitor the tonic muscle activity itself and visualize the level of activation, including visual feedback of tonic EMG displayed via a LED array, ${ }^{31}$ or small monitor, ${ }^{8}$ auditory feedback, ${ }^{32}$ or visualize head pressure by pushing the jaw against a blood pressure manometer with inflatable cuff. 19,33

The set points of the directly monitored tonic EMG levels used as a feed back to maintain the SCM muscle activation within specific levels, varied among researchers: $30-50 \mu \mathrm{V},{ }^{8} 40-150 \mu \mathrm{V},{ }^{34}$ and 50 $200 \mu \mathrm{V}^{35}$

It is recommend visual feedback to control tonic EMG activity as a routine technique to optimize c-VEMP amplitude and reproducibility.

\section{Out parameters}

\section{Latency}

Among others c-VEMP latencies vary with stimulus type (e.g. sound, galvanic, taps), amplitude, frequency, shape, duration and age. But as not all independent parameters are fully recognized and understood, and also identification of the peak on-set is done manually and arbitrary, it is best to establish the normal range of the latencies at each institution.

A significant delay of peak latencies P13 and N23 is considered pathological. The peak latencies P13 show a better reproducibility than that of $\mathrm{N} 23$

We advise to acquire adequate normative data of C-VEMP before using it as a diagnostic tool.

\section{Amplitude}

As not all independent parameters are fully recognized and understood, and because the identification of the peaks is done manually and arbitrary, it is best to establish the normal range of peak amplitudes or thresholds at each institution separately and not rely too much upon the data provided by the manufactory.

The c-VEMP response occurs as a stimulus synchronized reduction in tonic EMG activity. As the sacculo-collic reflex is inhibitory to the SCM contraction, c-VEMPs are therefore only detectable when the muscle is indeed contracted. The c-VEMPs amplitude therefore increases when the force exerted increase by recruitment of more motors units. C-VEMP amplitude also increases with a longer stimulus duration leading to a more prolonged and intense period of inhibition. The effect of both parameters would be an increase in the likelihood of a given motor unit being affected by the stimulus and thus a larger number of units to respond to each stimulus, evoking a larger response. ${ }^{35}$ Indeed, c-VEMP amplitude depends on the level of both the tonic SCM muscle activation and the stimulus intensity. ${ }^{21,26}$

The absolute c-VEMP amplitudes show a high inter-subject variability. Ochi et al., ${ }^{41}$ concluded that the $\mathrm{SD}$ of the C-VEMP amplitude was too large to be used in clinical evaluation, even when the amplitude was normalized with the absolute EMG values. ${ }^{37}$ Therefore c-VEMP amplitudes are in principle less appropriate for use in clinical practice. ${ }^{8,37}$

Nevertheless, many people used and still use the peak amplitudes because the total test duration is then shorter as compared to a threshold measurement. Some investigations show that amplitude asymmetries between the right and left ear stimulation could provide useful information in diagnosing audio vestibular and neurological disorders as well as in determine the likely side of pathology. ${ }^{38}$ The upper limit of the c-VEMP asymmetry ratio varies from $20 \%-36 \%$ in the literature..$^{24,27,36,37}$

\section{Thresholds}

The lowest stimulus intensity needed to evoke a c-VEMP response is called the threshold. They are all based on the fact that c-VEMP amplitudes increase with increasing stimulus intensity. The fundamental problem here is how to distinguish a small response from the background noise, which again is an arbitrary manual process.

VEMP thresholds in healthy subjects have been reported to range between $100-115 \mathrm{dBnHL},{ }^{9}$ and $75-105 \mathrm{dBnHL},{ }^{39}$ in response to click 
stimuli. For tone burst stimuli the thresholds ranges from 85-100 $\mathrm{dBnHL}$ at $1000 \mathrm{~Hz}(40)$ and from $60-75 \mathrm{dBnHL}$ at $250 \mathrm{~Hz}^{41}$

Although identifying c-VEMP thresholds seem to be a useful clinical approach for identifying peripheral vestibular diseases ${ }^{42,43}$ no literature was available to our knowledge at the start of our study in 2004 for normative data regarding application of the VEMP methodology for central diseases.

This application of threshold detection to quantify the VEMP responses is applied more widely, showing consistent and reproducible amplitudes and latencies. It is recommend the evaluation of VEMP thresholds and not VEMP amplitudes in investigations with central diseases.

\section{Conclusion}

More consensuses have been obtained regarding the precise stimulus and peak detection protocols for c-VEMP application in Otoneurology.

\section{Acknowledgments}

None.

\section{Conflicts of interest}

The author declares there are no conflicts of interest.

\section{References}

1. Colebatch JG. Vestibular evoked potentials. Curr Opin Neurol. 2001;14(1):21-26.

2. Colebatch JG, Halmagyi GM, Skuse NF. Myogenic potentials generated by a click-evoked vestibulocollic reflex. J Neurol Neurosurg Psychiatry.1994;57(2):190-197.

3. Zhou G, Cox LC. Vestibular evoked myogenic potentials: history and overview. Am J Audiol. 2004;13(2):135-143.

4. Huang TW, Su HC, Cheng PW. Effect of click duration on vestibularevoked myogenic potentials. Acta Otolaryngol.2005;125(2):141-144.

5. Welgampola MS, Colebatch JG. Characteristics and clinical applications of vestibular-evoked myogenic potentials. Neurology. 2005;64(10):1682-1688.

6. Curthoys IS, Vulovic V, Burgess AM, et al. The basis for using boneconducted vibration or air-conducted sound to test otolithic function. Ann N Y Acad Sci. 2011;12(33):231-241.

7. Saka N, Seo T, Fujimori K, et al. Vestibular-evoked myogenic potential in response to bone-conducted sound in patients with otosclerosis. Acta Otolaryngol. 2012;132(11):1155-1159.

8. Akin FW, Murnane OD, Panus PC, et al. The influence of voluntary tonic EMG level on the vestibular-evoked myogenic potential. J Rehabil Res Dev. 2004;41(3B):473-480.

9. Welgampola MS, Colebatch JG. Characteristics of tone burst-evoked myogenic potentials in the sternocleidomastoid muscles. Otol Neurotol. 2001;22(6):796-802.

10. Sheykholeslami K, Habiby Kermany M, Kaga K. Frequency sensitivity range of the saccule to bone-conducted stimuli measured by vestibular evoked myogenic potentials. Hear Res. 2001;160(1-2):58-62.

11. Murofushi T, Matsuzaki M, Wu CH. Short tone burst-evoked myogenic potentials on the sternocleidomastoid muscle: are these potentials also of vestibular origin? Arch Otolaryngol Head Neck Surg. 1999;125(6):660 664.
12. Rosengren SM, Govender S, Colebatch JG. The relative effectiveness of different stimulus waveforms in evoking VEMPs: significance of stimulus energy and frequency. $J$ Vestib Res. 2009;19(1-2):33-40.

13. McCue MP, Guinan JJ Jr. Spontaneous activity and frequency selectivity ofacoustically responsive vestibular afferents in the cat. $J$ Neurophysiol. 1995;74(4):1563-1572.

14. Herrmann BS, Guinan JJ, Rauch SD. Normalization reduces intersubject variability in cervical vestibular evoked myogenic potentials. Otol Neurotol. 2014;35(8):222-227.

15. Halmagyi GM, Colebatch JG, Curthoys IS. New tests of vestibular function. Baillieres Clin Neurol. 1994;3(3):485-500.

16. Tribukait A, Brantberg K, Bergenius J. Function of semicircular canals, utricles and saccules in deaf children. Acta Otolaryngol. 2004;124(1):41-48.

17. Bath AP, Harris N, Yardley MP. The vestibulo-collic reflex. Clin Otolaryngol Allied Sci. 1998;23(5):462-466.

18. Colebatch JG, Rothwell JC, Bronstein A, Click-evoked vestibular activation in the Tullio phenomenon. J Neurol Neurosurg Psychiatry. 1994;57(12):1538-1540.

19. Vanspauwen R, Wuyts FL, Van de Heyning PH. Improving vestibular evoked myogenic potential reliability by using a blood pressure manometer. Laryngoscope. 2006;116(1):131-135.

20. Al-Sebeih K, Zeitouni A. Vestibular-evoked myogenic potentials as a test of otolith function. Med Princ Pract. 2002;11(3):136-140.

21. Ferber Viart C, Soulier N, Dubreuil C. Cochleovestibular afferent pathways of trapezius muscle responses to clicks in human. Acta Otolaryngol. 1998;118(1):6-10.

22. Aidar RC, Suzuki FA. Vestibular evoked myogenic potential: new perspectives in multiple sclerosis. Braz $J$ Otorhinolaryngol. 2005;71(1):48-54

23. Akin FW, Murnane OD, Panus PC, et al. The influence of voluntary tonic EMG level on the vestibular-evoked myogenic potential. J Rehabil Res Dev. 2004;41(3B):473-480.

24. Basta D, Todt I, Ernst A. Normative data for P1/N1-latencies of vestibular evoked myogenic potentials induced by air- or bone-conducted tone bursts. Clin Neurophysiol. 2005;116(9):2216-2219.

25. Wang CT, Young YH. Comparison of the head elevation versus rotation methods in eliciting vestibular evoked myogenic potentials. Ear Hear. 2006;27(4):376-381.

26. Bhagat SP. Properties of binaural vestibular evoked myogenic potentials elicited with air-conducted and bone-conducted tone bursts. Int J Audiol. 2006;45(10):609-616.

27. Isaacson $\mathrm{B}$, Murphy $\mathrm{E}, \mathrm{Cohen} \mathrm{H}$. Does the method of sternocleidomastoid muscle activation affect the vestibular evoked myogenic potential response? J Vestib Res. 2006;16(4-5):187-191.

28. Monobe H, Murofushi T. Vestibular neuritis in a child with otitis media with effusion; clinical application of vestibular evoked myogenic potential by bone-conducted sound. Int $J$ Pediatr Otorhinolaryngol. 2004;68(11):1455-1458

29. Sheykholeslami K, Kaga K. The otolithic organ as a receptor of vestibular hearing revealed by vestibular-evoked myogenic potentials in patients with inner ear anomalies. Hear Res. 2002;165(1-2):62-67.

30. Rosengren SM, Govender S, Colebatch JG. The relative effectiveness of different stimulus waveforms in evoking VEMPs: significance of stimulus energy and frequency. $J$ Vestib Res. 2009;19(1-2):33-40.

31. Kingma CM, Wit HP. Asymmetric vestibular evoked myogenic 
potentials in unilateral Meniere patients. Eur Arch Otorhinolaryngol. 2011;268(1):57-61.

32. Rosengren SM, Kingma H. New perspectives on vestibular evoked myogenic potentials. Curr Opin Neurol. 2013;26(1):74-80.

33. Vanspauwen R, Wuyts FL, Van De Heyning PH. Validity of a new feedback method for the VEMP test. Acta Otolaryngol. 2006;126(8):796800 .

34. Maes L, Vinck BM, De Vel E, et al. The vestibular evoked myogenic potential: a test-retest reliability study. Clin Neurophysiol. 2009;120(3):594-600.

35. Wit HP, Kingma CM. A simple model for the generation of the vestibular evoked myogenic potential (VEMP). Clin Neurophysiol. 2006;117(6):1354-1358.

36. Colebatch JG, Halmagyi GM. Vestibular evoked potentials in human neckmuscles before and after unilateral vestibular deafferentation. Neurology. 1992;42(8):1635-1641.

37. Lim CL, Clouston P, Sheean G, et al. The influence of voluntary EMG activity and click intensity on the vestibular click evoked myogenic potential. Muscle Nerve. 1995;18(10):1210-1213.
38. Ochi K, Ohashi T, Nishino H. Variance of vestibular-evoked myogenic potentials. Laryngoscope. 2001;111(3):522-527.

39. Robertson DD, Ireland DJ. Vestibular evoked myogenic potentials. $J$ Otolaryngol. 1995;24(1):3-8.

40. Welgampola MS, Colebatch JG. Vestibulocollic reflexes: normal values and the effect of age. Clin Neurophysiol. 2001;112(11):1971-1979.

41. Ochi K, Ohashi T. Age-related changes in the vestibular-evoked myogenic potentials. Otolaryngol Head Neck Surg. 2003;129(6):655659

42. Zapala DA, Brey RH. Clinical experience with the vestibular evoked myogenic potential. J Am Acad Audiol. 2004;15(3):198-215.

43. Rauch SD, Zhou G, Kujawa SG, et al. Vestibular evoked myogenic potentials show altered tuning in patients with Ménière's disease. Otol Neurotol. 2004;25(3):333-338.

44. Lin MY, Timmer FC, Oriel BS, et al. Vestibular evoked myogenic potentials (VEMP) can detect asymptomatic saccular hydrops. Laryngoscope. 2006;116(6):987-992. 